\title{
mRCC: Unter Pazopanib länger ohne Symptome und Nebenwirkungen
}

\begin{abstract}
Krankheitssymptome und Therapienebenwirkungen beeinflussen die Lebensqualität von Krebspatienten. Im Hinblick auf diese Aspekte wurden nun die beiden Angiogenesehemmer Pazopanib und Sunitinib bei Patienten mit fortgeschrittenem oder metastasiertem Nierenzellkarzinom verglichen.
\end{abstract}

Zeit mit Toxizitäten vom Grad 3/4 (TOX), Zeit ohne Krankheitssymptome oder Toxizitäten vom Grad 3/4 und Zeit nach Tumorprogression oder -rezidiv. Die Summe der Zeiten aus den 3 Stufen (gewichtet nach Qualität) ergab die qualitätsadjustierte Zeit ohne Symptome oder Toxizität. Dieser Wert wurde in mehreren Analysen berechnet, wobei jeweils unterschiedliche Nutzwerte für die Zeiten mit Toxizitäten vom Grad 3/4 und die Zeiten nach Tumorprogression oder -rezidiv miteinander kombiniert wurden.

Die mediane Zeit mit Toxizitäten vom Grad 3/4 war unter Sunitinib im Vergleich zu Pazopanib um 31 Tage länger. Dieser Unterschied wurde jedoch durch die Reduktion der Zeit ohne Krankheits- symptome oder Toxizitäten vom Grad $3 / 4$ und die Zeit nach Tumorprogression oder -rezidiv wieder aufgehoben. Der Unterschied in der qualitätsadjustierten Zeit ohne Symptome oder Toxizität belief sich auf -11 Tage bis +43 Tage, fast immer zugunsten von Pazopanib. Typischerweise wurde ein signifikanter Unterschied dann beobachtet, wenn die Zeit ohne Krankheitssymptome oder Toxizitäten niedriger gewichtet wurde als die Zeit nach Progression oder Rezidiv.

Fazit: Die Patienten unter Pazopanib hatten eine etwas längere qualitätsadjustierte Zeit ohne Symptome oder Toxizität im Vergleich zu denen unter Sunitinib. Der Unterschied ging vor allem auf die kürzere Zeit mit Toxizitäten vom Grad 3/4 zurück.

Judith Neumaier

Beaumont JL et al. Quality-Adjusted Time Without Symptoms or Toxicity Analysis of Pazopanib Versus Sunitinib in Patients With Renal Cell Carcinoma. Cancer. 2016;122(7):1108-15.

\section{RCC: Individuelle Beobachtungsdauer nach der Nephrektomie}

\begin{abstract}
In dieser Studie wurde ein Modell untersucht, nach dem die Beobachtungsdauer von Patienten mit einem Nierenzellkarzinom (RCC) individuell in Abhängigkeit von verschiedenen Risikofaktoren berechnet wird.
\end{abstract}

D erzeit gibt es nur wenig Evidenz für die Leitlinien des National Comprehensive Cancer Network (NCCN) und der American Urological Association (AUA) zur Beobachtung von Patienten mit RCC nach radikaler oder partieller Nephrektomie. Den Ergebnissen einer großen Kohortenstudie zufolge bleibt sogar ein Drittel aller Rezidive unentdeckt, wenn die Leitlinien strikt befolgt werden, u. a. aufgrund einer zu kurzen Beobachtung. Individualisierte, risikoadaptierte Strategien sind daher erforderlich.

Die 2.511 Patienten aus dem Nephrektomie-Register der Mayo-Clinic mit nicht metastasiertem RCC hatten sich einer Operation unterzogen und wurden eingeteilt nach dem pathologischen Sta-
$\operatorname{dium}(\mathrm{pT} 1 \mathrm{Nx}-0, \mathrm{pT} 2 \mathrm{Nx}-0, \mathrm{pT} 3 / 4 \mathrm{Nx}-0$, pTjeglichesN1), der Rezidivlokalisation (Abdomen, Thorax, Knochen, andere), dem Alter $(<50,50-59,60-69,70-79$, $\geq 80$ Jahre) und dem Charlson-Komorbiditätsindex (CCI; $\leq 1$ und $\geq 2$ ).

676 Patienten hatten nach median 1,6 Jahren ein Rezidiv entwickelt, 435 waren nach median 1,1 Jahren an ihrem RCC gestorben. Nicht RCC-bedingte Todesfälle traten bei 615 Patienten nach median 6,1 Jahren auf. Anhand des Zeitpunkts, zu dem das Risiko für einen nicht RCC-bedingten Tod das Rezidivrisiko überstieg, wurden alters-, CCI-, stadien- und rezidivspezifische Beobachtungsdauern errechnet. Dabei ergaben sich stark unterschiedliche Zeiten:
Das Risiko für einen nicht RCC-bedingten Tod überstieg das für ein abdominelles Rezidiv bei Patienten, die einen Tumor im Stadium pT1Nx-0 und einen $\mathrm{CCI} \leq 1$ hatten, ab einem Alter von $\geq 80$ Jahre nach 6 Monaten, im Alter von 5059 Jahren erst nach 7 Jahren, bei Patienten unter 50 Jahren dagegen in mehr als 20 Jahren nie. Bei Patienten mit einem Tumor im Stadium pT1Nx-0 und einem $\mathrm{CCI} \geq 2$ überstieg das Risiko für einen nicht RCC-bedingten Tod das Risiko für ein abdominelles Rezidiv bereits 30 Tage nach der Operation.

Fazit: Der individuelle Ansatz berücksichtigt für die Entscheidung über die Beobachtungsdauer das Rezidivrisiko wie auch das Risiko für einen nicht RCCbedingten Tod. Die Strategie könnte die Balance zwischen Nutzen der Nachbeobachtung und Verteilung medizinischer Ressourcen verbessern. Judith Neumaier

Stewart-Merrill SB et al. Oncologic Surveillance After Surgical Resection for Renal Cell Carcinoma: A Novel Risk-Based Approach. J Clin Oncol. 2016;33(35):4151-7. 